\title{
GEOMETRIC INVARIANT THEORY
}

\author{
P. E. NEWSTEAD \\ CIMAT, GUANAJUATO, MEXICO, 27/28 NOVEMBER, 2006
}

In these lectures we will describe a method of constructing quotients in algebraic geometry. The natural context for the construction is that of schemes, but for simplicity we will work always with varieties defined over an algebraically closed field $k$; this field can have any characteristic. These notes are intended to be reasonably self-contained, although some proofs are omitted; further details, if needed, can be found in my Tata Institute notes

- P. E. Newstead, Introduction to moduli problems and orbit spaces, TIFR, Bombay, 1978 (out of print but many people have copies).

Other references are

- D. Mumford, J. Fogarty and F. Kirwan, Geometric invariant theory, 3rd. edition, Ergebnisse Math. 34, Springer-Verlag, Berlin, 1994 (the 1st. edition, published in 1965, is the "bible" on the subject)

- I. Dolgachev, Lectures on invariant theory, LMS Lecture Notes Series, 296, CUP, 2003

- R. P. Thomas, Notes on GIT and symplectic reduction for bundles and varieties, arXiv:math.AG/0512411 v3, 2006 (this is mainly useful for explaining the link between GIT and symplectic reduction, a topic which we shall not cover)

- P. E. Newstead, Vector bundles on algebraic curves (my "Polish" notes, available from http://www.mimuw.edu.pl/ jarekw/EAGER/lukecin02.html) (Lecture 1 in this series is similar to lecture 1 in the Polish notes and to a lecture given in the Boston/Tufts Algebraic Geometry Seminar, October 2006)

- S. Mukai, An introduction to invariants and moduli (translated by W. M. Oxbury), CUP 2003 (includes classical invariant theory as well as GIT and moduli of vector bundles on curves)

- J. Le Potier, Lectures on vector bundles (translated by A. Maciocia), CUP 1997 (includes a chapter on GIT) 


\section{Lecture 1 - Quotients}

1.1. Types of quotient. Let $G$ be an algebraic group acting rationally on a variety $X$ (that is, through a morphism $G \times X \rightarrow X:(g, x) \mapsto g \cdot x)$. Our object is to construct a good quotient for this action; what could this mean?

(i) Orbit space. It may happen that the set of orbits $X / G$ has a natural structure of variety. In fact this rarely happens, although there is always a dense Zariskiopen $G$-invariant subset $U$ of $X$ for which $U / G$ exists [Rosenlicht 1963] (a fact which is not as well known as it might be). However we are looking for something more global. Examples 1.1-1.3 below illustrate some of the problems with the orbit space approach.

(ii) Categorical quotient. We say that $\phi: X \rightarrow Y$ (or just $Y$ ) is a categorical quotient of $X$ by $G$ if every morphism $X \rightarrow Z$ which is constant on orbits factors uniquely through $\phi$. This is uniquely determined if it exists and has good functorial properties, but not necessarily good geometric ones (see the examples below). The ultimate in this approach is the use of stacks; these allow quotients to exist by definition, but geometrical properties may not be obvious.

Example 1.1. $k^{*}$ acts on $k^{2}$ by $\lambda .(x, y)=\left(\lambda x, \lambda^{-1} y\right)$. The orbits are

- for each $\alpha \in k^{*}$, the conic $\{(x, y): x y=\alpha\}$

- the punctured $x$-axis $\left\{(x, 0): x \in k^{*}\right\}$

- the punctured $y$-axis $\left\{(0, y): y \in k^{*}\right\}$

- the origin $\{(0,0)\}$.

In order to get a separated quotient, one has to combine the three last orbits listed and indeed one then gets a categorical quotient isomorphic to $k$, the quotient morphism being given by $(x, y) \mapsto x y$. One cannot obtain a separated orbit space (that is, as a variety), even if one deletes the orbit $\{(0,0)\}$, which has lower dimension than the others, since both punctured axes are limits of the other orbits as $\alpha \rightarrow 0$.

Example 1.2. $k^{*}$ acts on $k^{n}(n \geq 2)$ by $\lambda .\left(x_{1}, \ldots, x_{n}\right)=\left(\lambda x_{1}, \ldots, \lambda x_{n}\right)$. The origin lies in the closure of every orbit, so any morphism which is constant on orbits is constant. Thus there is no orbit space but there is a categorical quotient consisting of a single point.

Example 1.3. $k^{*}$ acts on $k^{n} \backslash\{(0,0)\}$ by the same formula. This time the projective space $\mathbb{P}^{n-1}$ is a categorical quotient and also an orbit space.

Our aim is to construct categorical quotients with good geometrical properties. Our method of doing this is Geometric Invariant Theory (GIT). There are other methods using stacks or algebraic spaces or by direct construction (Example 1.3 above, for instance) etc. Here we shall concentrate on GIT, which has proved extremely useful and, when $k$ is the complex numbers, has important and surprising connections with symplectic geometry. Proofs are not included; we will comment on these in Lecture 3.

We shall always write $G . x$ for the orbit $\{y \in X: y=g . x$ for some $g \in G\}$ of $x$. 
1.2. Rings of invariants. For any variety $X$, let $A(X)$ denote the algebra of morphisms $X \rightarrow k$. The action of $G$ on $X$ determines an action of $G$ on $A(X)$. We write $A(X)^{G}$ for the algebra of elements of $A(X)$ which are $G$-invariant. If $X \rightarrow Z$ is constant on orbits, the natural homomorphism $A(Z) \rightarrow A(X)$ has image contained in $A(X)^{G}$. In particular, if $Y$ is a categorical quotient, the homomorphism $A(Y) \rightarrow A(X)^{G}$ is an isomorphism.

Example 1.4. In Examples 1.1, 1.2, 1.3, we have $G=k^{*}$ and $A(X)^{G}=k[\alpha], k, k$ respectively. In Examples 1.1, 1.2, this gives good geometric answers, in Example 1.3, it does not. In fact, in Example 1.3, we have a very well behaved quotient $Y=\mathbb{P}^{n-1}$ and $A(Y)$ is isomorphic to $A(X)^{G}=k$, but this in no way represents the geometry of the quotient.

Example 1.5. $G=\mathrm{GL}(n)$ acts on $X=\mathrm{M}(n)(n \times n$ matrices $)$ by conjugation. It is not hard to see that $A(X)^{G}$ is the polynomial algebra on the coefficients of the characteristic polynomial. These coefficients determine a morphism $\mathrm{M}(n) \rightarrow k^{n}$, which is a categorical quotient, but not an orbit space since all matrices with the same eigenvalues map to the same point of $k$, but not all such matrices are in the same orbit (cf. Jordan normal forms).

1.3. Affine Quotients. Now suppose that $X$ is an affine variety and that we look for a quotient which is also an affine variety (this rules out Example 1.3, but includes Examples 1.1, 1.2 and 1.5). In this case $A(X)$ is just the affine coordinate ring of $X$, and, by Hilbert's Nullstellensatz, we can recover $X$ from $A(X)$. Moreover, a $k$-algebra has the form $A(Z)$ for some affine variety $Z$ if and only if it is finitely generated and has no nilpotent elements. The second property is necessarily true of $A(X)^{G}$, so one question to be answered is

Question. Is $A(X)^{G}$ finitely generated?

This is a version of Hilbert's 14th problem and the answer in general is NO [Nagata 1958]. However the answer is always YES if $G$ is reductive. The meaning of reductive will be discussed in Lecture 3; for the time being, it is enough to remark that many important examples, including $\mathrm{GL}(n), \mathrm{SL}(n)$ and $\mathrm{PGL}(n)$, are reductive. It turns out that this is sufficient for us to construct well behaved quotients in the affine case.

Theorem 1.6. Let $G$ be a reductive group acting on an affine variety $X$. Then there exists an affine variety $Y$ and a morphism $\phi: X \rightarrow Y$ such that

(i) $\phi$ is constant on orbits;

(ii) $\phi$ is surjective;

(iii) if $U$ is a Zariski-open subset of $Y$, the induced homomorphism

$$
\phi^{*}: A(U) \rightarrow A\left(\phi^{-1}(U)\right)^{G}
$$

is an isomorphism;

(iv) if $W$ is a Zariski-closed $G$-invariant subset of $X$, then $\phi(W)$ is closed in $Y$;

(v) if $W_{1}, W_{2}$ are Zariski-closed $G$-invariant subsets of $X$ and $W_{1} \cap W_{2}=\emptyset$, then $\phi\left(W_{1}\right) \cap \phi\left(W_{2}\right)=\emptyset$. 
Corollary 1.7. For any Zariski-open subset $U$ of $Y, U$ is a categorical quotient of $\phi^{-1}(U)$ by $G$.

Corollary 1.8. If $\phi\left(x_{1}\right)=\phi\left(x_{2}\right)$, then $\overline{G \cdot x_{1}} \cap \overline{G \cdot x_{2}} \neq \emptyset$.

Corollary 1.9. If $G$ acts on $\phi^{-1}(U)$ with closed orbits, then $U=\phi^{-1}(U) / G$ (i.e. $U$ is an orbit space).

Note that Corollary 1.8 states that $\phi$ separates orbits to the greatest extent possible, consistent with $Y$ being a variety. This is perhaps more significant than the simple fact that $A(X)^{G}$ is finitely generated.

1.4. Projective quotients. The case in which we are most interested is when $X$ is projective; this is because we would like our quotients to be complete (compact) or at least to have natural compactifications. To apply the previous section, we need to consider affine open subsets of $X$ which are invariant under $G$. We suppose $X \subset \mathbb{P}^{n}$ and that $G$ acts linearly, that is through a representation of $G$ in $G L(n+1)$. Then $G$ acts on the polynomial ring $k\left[X_{0}, \ldots, X_{n}\right]$. If $f$ is any non-constant $G$-invariant homogeneous polynomial for this action, then $X_{f}=\{x \in X: f(x) \neq 0\}$ is a $G$ invariant affine open subset of $X$.

Definition. A point $x \in X$ is

- semistable for the action of $G$ if there exists $f$ such that $x \in X_{f}$;

- stable for the action of $G$ if $x$ has finite stabiliser (or equivalently $\operatorname{dim} G . x=$ $\operatorname{dim} G$ ) and there exists $f$ as above such that $G$ acts on $X_{f}$ with closed orbits. (In GIT, this is called "properly stable".)

Lemma 1.10. The subsets $X^{s s}, X^{s}$ of $X$ of semistable (resp. stable) points of $X$ are $G$-invariant Zariski-open subsets of $X$.

Exercise. Prove Lemma 1.10.

Definition. $\phi: X \rightarrow Y$ is

- a good quotient of $X$ by $G$ if $\phi$ is an affine morphism (that is, the inverse image of every affine Zariski-open set in $Y$ is affine) and (i)-(v) of Theorem 1.6 hold;

- a geometric quotient if it is a good quotient and also an orbit space.

It is conventional to write $Y=X / / G$ when $Y$ is a good quotient and $Y=X / G$ when $Y$ is a geometric quotient. The concept of "good quotient" given here is due to Seshadri; the idea originates in GIT, but the definition given there is not identical with ours. Our "geometric quotient" differs from that in GIT by the insistence that $\phi$ be an affine morphism.

The properties of the corollaries to Theorem 1.6 carry over to good quotients.

Proposition 1.11. Let $\phi: X \rightarrow Y$ be a good quotient of $X$ by $G$. Then

(i) $Y$ is a categorical quotient of $X$ by $G$;

(ii) $\phi\left(x_{1}\right)=\phi\left(x_{2}\right) \Leftrightarrow \overline{G \cdot x_{1}} \cap \overline{G \cdot x_{2}} \neq \emptyset$; 
(iii) if the action of $G$ on $X$ is closed, then $Y=X / G$ is a geometric quotient of $X$ by $G$.

We come now to the main theorem of this lecture.

Theorem 1.12. Let $G$ be a reductive group acting linearly on a projective variety $X$. Then

(i) there exists a good quotient $\phi: X^{s s} \rightarrow Y$ and $Y=X^{s s} / / G$ is projective;

(ii) there exists a Zariski-open subset $Y^{s}$ of $Y$ such that $\phi^{-1}\left(Y^{s}\right)=X^{s}$ and $Y^{s}=$ $X^{s} / G$ is a geometric quotient of $X^{s}$;

(iii) for $x_{1}, x_{2} \in X^{s s}, \phi\left(x_{1}\right)=\phi\left(x_{2}\right) \Leftrightarrow \overline{G \cdot x_{1}} \cap \overline{G \cdot x_{2}} \cap X^{s s} \neq \emptyset$;

(iv) for $x \in X^{s s}, x$ is stable if and only if $x$ has finite stabiliser and $G . x$ is closed in $X^{s s}$.

Remark 1.13. In some ways the most significant point of this theorem is that $Y$ is projective. This means both that it is complete and that it can be embedded in projective space.

Remark 1.14. For $G$ not necessarily reductive, Rosenlicht's result gives a dense Zariski-open subset $U$ of $X$ such that $U / G$ exists with all the properties of a geometric quotient except that the morphism $\phi$ need not be affine. 


\section{Lecture 2 - The Hilbert-Mumford CRITERION}

It is in general a very difficult problem to calculate a ring of invariants. However, if we are simply interested in calculating basic properties of a projective quotient, it is often sufficient to compute the stable and semistable points for the action. In this lecture we will describe a usable criterion for doing this, due originally to Hilbert for semistability for $G=\operatorname{SL}(n)$ and extended by Mumford to stability and arbitrary $G$. We begin with a definition of stability not involving projective space. We suppose throughout that $G$ is a reductive group.

\subsection{Affine definition of stability.}

Proposition 2.1. Let $X$ be a projective variety in $\mathbb{P}^{n}$ and $G$ a reductive group acting linearly on $X$. Let $x \in X$ and $\hat{x} \in k^{n+1}$ be a point lying over $x$. Then

(i) $x$ is semistable if and only if $0 \notin \overline{G . \hat{x}}$;

(ii) $x$ is stable if and only if the stabiliser of $\hat{x}$ is finite and $G . \hat{x}$ is closed in $k^{n+1}$.

Proof. (i) Suppose first that $x$ is semistable, and let $f$ be a $G$-invariant homogeneous polynomial of degree $\geq 1$ such that $f(x) \neq 0$. We have clearly $f(\hat{x}) \neq 0$, so $f(y)$ is equal to a non-zero constant for $y \in G$. $\hat{x}$. Hence $0 \notin \overline{G . \hat{x}}$.

Conversely, if $0 \notin \overline{G . \hat{x}}$, it is a simple consequence of geometric reductivity (see Lemma 3.3 in my Tata Institute notes) that there exists an invariant polynomial $f$ such that

$$
f(0)=0, \quad f(\overline{G . \hat{x}})=1 .
$$

But then $f$ has constant term 0 ; so some homogeneous part of $f$ must be non-zero at $\hat{x}$. Hence $x$ is semistable.

(ii) Suppose that $x$ is stable. Then $x$ has finite stabiliser and there exists a $G$ invariant homogeneous polynomial $f$ of degree $\geq 1$ such that $f(x) \neq 0$ and $G . x$ is closed in $X_{f}$. It is obvious that $\hat{x}$ has finite stabiliser. Now put $\alpha=f(\hat{x}) \neq 0$ and let

$$
Z_{\alpha}=\left\{y \in k^{n+1}: f(y)=\alpha\right\} .
$$

Clearly $Z_{\alpha}$ is closed in $k^{n+1}$ and it is easy to see that the morphism $Z_{\alpha} \rightarrow \mathbb{P}_{f}^{n}$ is surjective and finite. Moreover the inverse image of $G . x$ is closed in $Z_{\alpha}$ and is the union of a finite number of orbits of $G$ acting on $Z_{\alpha}$. Since all these orbits have the same dimension, each of them, in particular $G . \hat{x}$, is closed in $Z_{\alpha}$ and therefore in $k^{n+1}$.

Conversely suppose that $\hat{x}$ has finite stabiliser and $G . \hat{x}$ is closed in $k^{n+1}$. By (i), $x$ is semistable, so there exists a $G$-invariant homogeneous polynomial $f$ of degree $\geq 1$ such that $f(x) \neq 0$. As above $Z_{\alpha} \rightarrow \mathbb{P}_{f}^{n}$ is surjective and finite. This morphism maps $G$. $\hat{x}$ surjectively to G.x; it follows that $x$ has finite stabiliser and G.x is closed in $\mathbb{P}_{f}^{n}$. This holds for all $f$ such that $f(x) \neq 0$, so G.x is closed in $X^{s s}$. It follows from Theorem $1.12($ iv) that $x$ is stable.

2.2. Actions of $k^{*}$. Suppose first that $G=k^{*}$. We identify $k^{*}$ with a subset of $\mathbb{P}^{1}$ in the obvious way. In fact, for any $\alpha \in k$, we identify $\alpha$ with $(1, \alpha) \in \mathbb{P}^{1}$ and write $\infty$ for the extra point $(0,1)$. Note that any morphism $k^{*} \rightarrow \mathbb{P}^{n}$ extends to a morphism 
$\mathbb{P}^{1} \rightarrow \mathbb{P}^{n}$, but the corresponding statement is not true for a morphism $k^{*} \rightarrow k^{n+1}$. In fact, if $\phi$ is such a morphism and is non-constant,

(a) any point of $\overline{\phi\left(k^{*}\right)} \backslash \phi\left(k^{*}\right)$ must be equal to one of $\lim _{t \rightarrow 0} \phi(t)$ and $\lim _{t \rightarrow \infty} \phi(t)$;

(b) $\phi\left(k^{*}\right)$ is closed in $k^{n+1}$ if and only if neither limit exists.

Now suppose $G=k^{*}$ acts linearly on a projective variety $X$ in $\mathbb{P}^{n}$. The induced linear action on $k^{n+1}$ can be diagonalised. In other words there exists a basis $e_{0}, \ldots, e_{n}$ of $k^{n+1}$ such that

$$
t . e_{i}=t^{r_{i}} e_{i}
$$

for some integers $r_{i}$. Now choose $\hat{x} \in k^{n+1}$ lying over $x$ and write $\hat{x}=\sum \hat{x}_{i} e_{i}$, so that

$$
t . \hat{x}=\sum t^{r_{i}} \hat{x}_{i} e_{i}
$$

and define

$$
\mu(x)=\max \left\{-r_{i}: x_{i} \neq 0\right\} .
$$

Thus $\mu(x)$ is the unique integer $\mu$ such that $\lim _{t \rightarrow 0} t^{\mu}(t . \hat{x})$ exists and $\neq 0$. (It is easy to see that $\mu(x)$ is independent of the choice of $\hat{x}$ and of the basis $e_{0}, \ldots, e_{n}$.) Note that

$$
\begin{gathered}
\mu(x)>0 \Leftrightarrow \lim _{t \rightarrow 0} t . \hat{x} \text { does not exist, } \\
\mu(x)=0 \Leftrightarrow \lim _{t \rightarrow 0} t . \hat{x} \text { exists and } \neq 0 .
\end{gathered}
$$

Similarly we define

$$
\mu^{-}(x)=\max \left\{r_{i}: x_{i} \neq 0\right\}
$$

Then

$$
\begin{aligned}
\mu^{-}(x)>0 \Leftrightarrow \lim _{t \rightarrow \infty} t . \hat{x} \text { does not exist, } \\
\mu^{-}(x)=0 \Leftrightarrow \lim _{t \rightarrow \infty} t . \hat{x} \text { exists and } \neq 0 .
\end{aligned}
$$

The following proposition now follows immediately from Proposition 2.1, (a) and (b).

Proposition 2.2. Let $G=k^{*}$ act linearly on a projective variety $X$ in $\mathbb{P}^{n}$. Then, for any $x \in X$,

(i) $x$ is semistable if and only if $\mu(x) \geq 0$ and $\mu^{-}(x) \geq 0$;

(ii) $x$ is stable if and only if $\mu(x)>0$ and $\mu^{-}(x)>0$.

2.3. The criterion. We now consider an arbitrary linear action of a reductive group $G$ on a projective variety $X$.

Definition A 1-parameter subgroup (1-PS) of $G$ is a non-trivial homomorphism of algebraic groups $\lambda: k^{*} \rightarrow G$.

It is a standard fact that the image of any homomorphism of algebraic groups is closed. It now follows easily from Proposition 2.1 that a stable (semistable) point of $X$ for the action of $G$ is necessarily stable (semistable) for the action of $k^{*}$ induced by any 1-PS.

For any 1-PS $\lambda$, we write $\mu(x, \lambda)$ for the value of $\mu(x)$ for the action of $k^{*}$ on $X$ induced by $\lambda$. (It is not necessary to define $\mu^{-}(x, \lambda)$ because this is just $\mu\left(x, \lambda^{-1}\right)$ 
where $\lambda^{-1}$ is the 1-PS defined by $\lambda^{-1}(t)=\lambda\left(t^{-1}\right)$.) It now follows from Proposition 2.2 that

- $x$ semistable $\Rightarrow \mu(x, \lambda) \geq 0$ for every 1-PS $\lambda$ of $G$;

- $x$ stable $\Rightarrow \mu(x, \lambda)>0$ for every 1-PS $\lambda$ of $G$.

We can now state the Hilbert-Mumford Criterion.

Theorem 2.3. Let $G$ be a reductive group acting linearly on a projective variety $X$ in $\mathbb{P}^{n}$. Then the two $\Rightarrow$ above can be replaced by $\Leftrightarrow$.

In order to prove this theorem, one has to prove the following assertions.

- $x$ non-stable $\Rightarrow \exists \lambda$ such that $\mu(x, \lambda) \leq 0$,

- $x$ non-semistable $\Rightarrow \exists \lambda$ such that $\mu(x, \lambda)<0$.

In view of Proposition 2.2, this is equivalent to showing that, if $x$ is non-stable (nonsemistable), then there is a 1-PS $\lambda$ such that $x$ is still non-stable (non-semistable) for the induced action of $k^{*}$. These statements are at least plausible if $G$ has a plentiful supply of 1-PS; this is the significance of the hypothesis that $G$ is reductive.

A proof for the semistable case when $G=\mathrm{SL}(m)$ and $k=\mathbb{C}$ was given by Hilbert using convergent power series. Mumford and Seshadri obtained a proof valid for all $k$ and all reductive $G$ using formal power series and a theorem of Iwahori. A proof for all $k$ but restricted to the case $G=\mathrm{SL}(m)$ is given in my Tata Institute notes and also in Mukai's book.

2.4. An observation. The following remark can simplify computations.

Remark 2.4. It follows at once from the definition that

$$
\mu(g . x, \lambda)=\mu\left(x, g^{-1} \lambda g\right)
$$

for any $g \in G$. This allows us to replace $\lambda$ by a convenient conjugate in making calculations. In particular, for $g=\operatorname{SL}(n)$, it is well known that every 1-PS is conjugate to a diagonal matrix of the form

$$
\lambda(t)=\left(\begin{array}{cccc}
t^{r_{0}} & 0 & \cdots & 0 \\
0 & t^{r_{1}} & \cdots & 0 \\
\vdots & \vdots & \ddots & \vdots \\
0 & \cdots & \cdots & t^{r_{n-1}}
\end{array}\right)
$$

with

$$
\sum r_{i}=0, \quad r_{0} \geq r_{1} \geq \cdots \geq r_{n-1}, \quad \text { not all } r_{i}=0 .
$$

(This follows almost at once from the fact (already used) that any linear action of $k^{*}$ is diagonalisable.) We deduce

Proposition 2.5. Let $\mathrm{SL}(n)$ act linearly on a projective variety $X$. A point $x$ of $X$ is stable (semistable) for this action if and only if $\mu(g . x, \lambda)>0(\geq 0)$ for every $g \in \operatorname{SL}(n)$ and every 1-PS $\lambda$ of $\mathrm{SL}(n)$ of the form (1). 


\section{LeCture 3 - Further Details}

3.1. Reductivity and finite generation. The key to the proofs of the results in Lecture 1 is the concept of a reductive group.

Note first that any linear algebraic group $G$ possesses a unique maximal connected normal solvable subgroup, called its radical.

Definition. Let $G$ be a linear algebraic group. Then $G$ is

- reductive if its radical is isomorphic to a direct product of copies of $k^{*}$;

- linearly reductive if, for every linear action of $G$ on $k^{n}$, and every invariant point $v$ of $k^{n}, v \neq 0$, there exists a $G$-invariant homogeneous polynomial $f$ of degree 1 such that $f(v) \neq 0$;

- geometrically reductive if, for every linear action of $G$ on $k^{n}$, and every invariant point $v$ of $k^{n}, v \neq 0$, there exists a $G$-invariant homogeneous polynomial $f$ of degree $\geq 1$ such that $f(v) \neq 0$.

Warning. Although these terms are now widely accepted, many different terms have been used in the past.

It is easy to see that the groups $\operatorname{GL}(n), \operatorname{SL}(n)$ and $\operatorname{PGL}(n)$ are all reductive, also $\left(k^{*}\right)^{m}$ for any positive integer $m$, but not $k$ as an additive group. It is not difficult to see that every geometrically reductive group is reductive; and, in characteristic 0 , it is a theorem of Weyl that every reductive group is linearly reductive (so in this case all three conditions coincide). In characteristic $p$, however, there are very few linearly reductive groups; certainly $\operatorname{GL}(n), \operatorname{SL}(n)$ and $\operatorname{PGL}(n)$ are not linearly reductive for $n \geq 2$.

The link between reductivity and finite generation is provided by

Nagata's Theorem. Let $G$ be a geometrically reductive group acting rationally on a finitely generated $k$-algebra $R$. Then $R^{G}$ is finitely generated.

In order to justify our assertions in Lecture 1, we need also

Mumford's Conjecture. Every reductive group is geometrically reductive.

This was conjectured by Mumford in the 1st. edition of GIT and proved by Haboush in 1974. In fact, geometric reductivity is the key not only to finite generation but also to establishing the geometric properties in Theorem 1.6.

Remark 3.1. (i) It is possible for $A(X)^{G}$ to be finitely generated even when $G$ is not reductive. Much work has been done on the extension of GIT to non-reductive groups.

(ii) It is possible for a categorical quotient to exist even if $A(X)^{G}$ is not finitely generated.

(iii) As already noted (Example 1.3), the finite generation of $A(X)^{G}$ does not imply that the corresponding affine variety is even a categorical quotient.

3.2. Proof of Theorem 1.6. The hypotheses of Theorem 1.6 imply, by Nagata's Theorem, that $A(X)^{G}$ is a finitely generated $k$-algebra; moreover it is a subalgebra of $A(X)$ and therefore has no nilpotent elements. Let $Y$ be the corresponding affine 
variety, and $\phi$ the morphism corresponding to the inclusion $A(X)=A(X)^{G} \subset A(X)$. We prove that $Y$ and $\phi$ have the required properties.

(i) Suppose that there exist $g \in G, x \in X$ with $\phi(g . x) \neq \phi(x)$. Then, since $Y$ is affine, there exists $f \in A(Y)$ such that

$$
f(\phi(g . x)) \neq f(\phi(x)) .
$$

This contradicts the fact that $f \in A(X)^{G}$.

(ii) Let $y \in Y$ and let $f_{1}, \ldots, f_{r}$ generate the maximal ideal in $A(Y)=A(X)^{G}$ corresponding to the point $y$. It is not difficult to prove that

$$
\sum f_{i} A(X) \neq A(X)
$$

(see my Tata Institute notes Lemma 3.4.2). Hence there exists a maximal ideal of $A(X)$ containing $\sum f_{i} A(X)$. If $x$ is the point of $X$ corresponding to this maximal ideal, then we have $f_{i}(x)=0$ for all $i$; so $\phi(x)=y$.

(iii) First note that it is sufficient to prove (iii) for the open sets $U$ of any fixed basis. Hence we can suppose that $U=Y_{f}$ for some non-zero $f \in A(Y)=A(X)^{G}$. In this case, $\phi^{-1}(U)=X_{f}$; so we require to prove that

$$
\left(A(X)^{G}\right)_{f}=\left(A(X)_{f}\right)^{G}
$$

for any $f \in A(X)^{G}$, where

$$
A(X)_{f}=A\left(X_{f}\right)=\left\{h / f^{r}: r \geq 0, h \in A(X)\right\} .
$$

This is easy to verify directly.

(iv) and (v). For any two disjoint closed $G$-invariant subsets $W_{1}, W_{2}$ of $X$, there exists (Lemma 3.3 in my Tata Institute notes) an element $f \in A(X)^{G}$ such that

$$
f\left(W_{1}\right)=0, \quad f\left(W_{2}\right)=1 .
$$

Regarding $f$ as an element of $A(Y)$, we obtain from this

$$
f\left(\phi\left(W_{1}\right)\right)=0, \quad f\left(\phi\left(W_{2}\right)\right)=1 ;
$$

hence

$$
\overline{\phi\left(W_{1}\right)} \cap \overline{\phi\left(W_{2}\right)}=\emptyset
$$

This proves (v); moreover, if $W$ is a closed $G$-invariant subset of $X$ and $y \in \overline{\phi(W)} \backslash$ $\phi(W)$, we can apply the above result with $W_{1}=W, W_{2}=\phi^{-1}(y)$ to get a contradiction.

3.3. Proof of Corollaries 1.7, 1.8, 1.9, Proposition 1.11. The proofs of the corollaries use only the results of Theorem 1.6, hence also give proofs of Proposition 1.11. The only difficult proof is that of Corollary 1.7, the basic reason being that, even for $U=Y$, one has to prove the universal property for morphisms into any variety, not just into an affine variety. A detailed proof is contained in my Tata Institute notes (pp64, 65). The fact that the result extends to any open set $U$ of $Y$ requires the following lemma, which is a useful extension of Theorem 1.6(v). 
Lemma 3.2. Let $X, G, Y, \phi$ be as in Theorem 1.6, and let $U$ be an open subset of $Y$. If $W_{1}, W_{2}$ are disjoint $G$-invariant subsets of $\phi^{-1}(U)$ and are closed in $\phi^{-1}(U)$, then

$$
\phi\left(W_{1}\right) \cap \phi\left(W_{2}\right)=\emptyset .
$$

Proof. Let $\overline{W_{i}}$ denote the closure of $W_{i}$ in $X$, and suppose that $y \in \phi\left(W_{1}\right) \cap \phi\left(W_{2}\right)$. Then $\phi^{-1}(y) \cap \overline{W_{1}}$ and $\overline{W_{2}}$ are closed $G$-invariant subsets of $X$ whose images under $\phi$ both contain $y$. Hence by Theorem $1.6(\mathrm{v})$

$$
\phi^{-1}(y) \cap \overline{W_{1}} \cap \overline{W_{2}} \neq \emptyset .
$$

But $W_{1}$ and $W_{2}$ are closed in $\phi^{-1}(U)$; so

$$
\phi^{-1}(U) \cap \overline{W_{1}} \cap \overline{W_{2}}=W_{1} \cap W_{2}=\emptyset .
$$

This gives the required contradiction.

The other two proofs are straightforward.

Proof of Corollary 1.8. Take $W_{i}=\overline{G \cdot x_{i}}$ in Theorem 1.6(v).

Proof of Corollary 1.9. We need to show that, if $x_{1}, x_{2} \in \phi^{-1}(U)$ do not belong to the same orbit, then $\phi\left(x_{1}\right) \neq \phi\left(x_{2}\right)$. This follows at once by taking $W_{i}=G \cdot x_{i}$ in Lemma 3.2 .

3.4. Proof of Theorem 1.12. (i) We begin by recalling some general facts about projective varieties and graded $k$-algebras.

Let $\hat{X}$ denote the affine variety in $k^{n+1}$ lying over $X$; for each homogeneous element $f$ of $A(\hat{X})$ of degree $\geq 1$ we have an affine open subset $X_{f}=\{x \in X: f(x) \neq 0\}$ of $X$. Let $R$ be a finitely generated homogeneous subalgebra of $A(\hat{X})$ and write $X_{R}$ for the union of those $X_{f}$ for which $f \in R$. Then there exists a projective variety $Y$ (usually denoted by $\operatorname{Proj} R$ ) and a morphism $\phi: X_{R} \rightarrow Y$ such that

(a) $Y$ is covered by affine open sets $Y_{f}$, one for each homogeneous element $f$ of $R$ of degree $\geq 1$, and $A\left(Y_{f}\right)$ is isomorphic to the algebra

$$
\left(R_{f}\right)_{0}=\left\{h / f^{r}: r \geq 0, h \in R \text { homogeneous of degree } r(\operatorname{deg} f)\right\} ;
$$

(b) $\phi^{-1}\left(Y_{f}\right)=X_{f}$ and $\phi: X_{f} \rightarrow Y_{f}$ is the morphism corresponding to the inclusion of $\left(R_{f}\right)_{0}$ in $A\left(X_{f}\right)=\left(A(\hat{X})_{f}\right)_{0}$.

In our situation, note that the linear action of $G$ on $X$ induces an action on $A(\hat{X})$ which preserves the degree of every homogeneous element. Hence $R=A(\hat{X})^{G}$ is a homogeneous subalgebra of $A(\hat{X})$, and it is also finitely generated by Nagata's Theorem. Let $Y$ and $\phi$ be as constructed above, and note that $X_{R}=X^{s s}$. By Theorem 1.6 and (b) above, together with the fact that $\left(\left(A(\hat{X})^{G}\right)_{f}\right)_{0}=\left(\left(A(\hat{X})_{f}\right)_{0}\right)^{G}$, it follows that $\left(Y_{f}, \phi\right)$ is a good quotient of $X_{f}$ for every homogeneous element $f$ of $A(\hat{X})^{G}$ of degree $\geq 1$. It follows easily that $(Y, \phi)$ is a good quotient of $X^{s s}$. 
(ii) Put $Y^{s}=\phi\left(X^{s}\right)$ and let $Y^{0}$ be the union of those $Y_{f}$ for which the action of $G$ on $X_{f}$ is closed. Clearly $X^{s} \subset \phi^{-1}\left(Y^{0}\right)$ and so $Y^{s} \subset Y^{0}$. Now by Proposition 1.11, $\left(Y^{0}, \phi\right)$ is a geometric quotient of $X^{0}=\phi^{-1}\left(Y^{0}\right)$. It follows that $X^{s}=\phi^{-1}\left(Y^{s}\right)$ and

$$
Y^{0} \backslash Y^{s}=\phi\left(X^{0} \backslash X^{s}\right)
$$

Hence $Y^{0} \backslash Y^{s}$ is closed in $Y^{0}$ by condition (iv) for a good quotient, and so $Y^{s}$ is open in $Y^{0}$ and therefore also in $Y$. It follows that $\left(Y^{s}, \phi\right)$ is a good quotient of $X^{s}$ and hence a geometric quotient.

(iii) follows at once from Proposition 1.11(ii).

(iv) If $x \in X^{s}$, then

$$
\phi^{-1}(\phi(x)) \subset \phi^{-1}\left(Y^{s}\right)=X^{s} .
$$

Since $\phi^{-1}(\phi(x))$ is closed in $X^{s s}$, it follows that

$$
\overline{G . x} \cap X^{s s} \subset X^{s} .
$$

But the action of $G$ on $X^{s}$ is closed by (ii); hence $G . x$ is closed in $X^{s}$ and therefore also in $X^{s s}$.

For the converse, note that, since $x \in X^{s s}$, there exists a $G$-invariant homogeneous polynomial $f$ of degree $\geq 1$ such that $x \in X_{f}$. If $G . x$ is closed in $X^{s s}$, then certainly $G . x$ is closed in $X_{f}$. It follows easily from this, and the fact that $x$ has finite stabiliser, that $x$ is stable (see Lemma 3.15 of my Tata Institute notes for details).

3.5. Linearisation. The ideas of Lecture 1 can be slightly generalised and the definitions of stability and semistability placed in a more natural context. The point is that, if we are given an action of an algebraic group $G$ on a variety $X$, there may be many ways of embedding $X$ in a projective space and linearising the action of $G$.

So let $X$ be any variety, $G$ an algebraic group acting rationally on $X$, and $L$ a line bundle over $X$; we denote by $p: L \rightarrow X$ the natural projection. A linearisation of the action of $G$ with respect to $L$ is an action of $G$ on $L$ such that

(i) for all $y \in L, g \in G$,

$$
p(g \cdot y)=g \cdot p(y)
$$

(ii) for all $x \in X, g \in G$, the map

$$
L_{x} \rightarrow L_{g . x}: y \mapsto g . y
$$

is linear.

An $L$-linear action of $G$ on $X$ is an action of $G$ together with a linearisation of this action with respect to $L$.

This concept allows us to generalise the definitions of stable and semistable. Note first that an $L$-linear action of $G$ on $X$ determines an action on the space of sections of any tensor power $L^{r}$ of $L$. Moreover, for any $G$-invariant section $f$ of $L^{r}$,

$$
X_{f}=\{x \in X: f(x) \neq 0\}
$$

is an open set of $X$, invariant under $G$.

Definitions. Let $X$ be a variety and $L$ a line bundle over $X$. For any $L$-linear action of a reductive group $G$ on $X$, a point $x$ is called 
- semistable if, for some positive integer $r$, there exists a $G$-invariant section $f$ of $L^{r}$ such that $f(x) \neq 0$ and $X_{f}$ is affine;

- stable if $x$ has finite stabiliser and, for some positive integer $r$, there exists a $G$-invariant section $f$ of $L^{r}$ such that $f(x) \neq 0, X_{f}$ is affine and the action of $G$ on $X_{f}$ is closed.

We write $X^{s s}\left(X^{s}\right)$ for the set of semistable (stable) points, the dependence on the linearisation of the action of $G$ being understood.

Example 3.3. Let $X$ be a projective variety in $\mathbb{P}^{n}$ and $L$ the restriction to $X$ of the hyperplane bundle $\mathcal{O}(1)$. Any linear action of $G$ on $X$ then induces an $L$-linear action, so both sets of definitions make sense. Fortunately, it is easy to prove that, in this situation,

$$
X^{s s}(L)=X^{s s} \text { and } X^{s}(L)=X^{s} .
$$

Theorem 1.12 extends to our new situation and gives

Theorem 3.4. Let $X$ be a variety and $L$ a line bundle over $X$. Then, for any L-linear action of a reductive group $G$ on $X$,

(i) there exists a good quotient $\phi: X^{s s}(L) \rightarrow Y$ and $Y=X^{s s}(L) / / G$ is quasiprojective;

(ii) there exists a Zariski-open subset $Y^{s}$ of $Y$ such that $\phi^{-1}\left(Y^{s}\right)=X^{s}(L)$ and $Y^{s}=X^{s}(L) / G$ is a geometric quotient of $X^{s}(L)$;

(iii) for $x_{1}, x_{2} \in X^{s s}(L), \phi\left(x_{1}\right)=\phi\left(x_{2}\right) \Leftrightarrow \overline{G \cdot x_{1}} \cap \overline{G \cdot x_{2}} \cap X^{s s}(L) \neq \emptyset$;

(iv) for $x \in X^{s s}(L), x$ is stable if and only if $x$ has finite stabiliser and $G . x$ is closed in $X^{s s}(L)$.

Note that no assumption of projectivity (or quasi-projectivity) is made on $X$. The fact that $Y$ is quasi-projective depends on the fact that $X^{s s}(L)$ is quasi-projective. This in turn depends on the concept of an ample line bundle (one for which some power $L^{r}$ determines an embedding in a projective space) and the fact that the restriction of $L$ to $X^{s s}(L)$ is always ample. 


\section{LECTURE 4 - EXAMPLES}

\subsection{An elementary example.}

Example 4.1. Let $k^{*}$ act on $\mathbb{P}^{n}(n \geq 2)$ by

$$
\lambda .\left(x_{0}: \ldots: x_{n}\right)=\left(\lambda x_{0}: \ldots, \lambda x_{n-1}: \lambda^{-n} x_{n}\right) .
$$

Writing $x=\left(x_{0}: \ldots: x_{n}\right)$, we have

$$
\mu(x)= \begin{cases}n & \text { if } x_{n} \neq 0 \\ -1 & \text { if } x_{n}=0\end{cases}
$$

and

$$
\mu^{-}(x)= \begin{cases}1 & \text { if } x_{0}, \ldots, x_{n-1} \text { are not all } 0 \\ -n & \text { if } x_{0}=\ldots=x_{n-1}=0 .\end{cases}
$$

So, by Proposition 2.2,

- $x$ is stable if $x_{n} \neq 0$ and $x_{0}, \ldots, x_{n-1}$ are not all 0 ;

- $x$ is not semistable if $x=\left(x_{1}: \ldots: x_{n-1}: 0\right)$ or $x=(0: \ldots: 0: 1)$.

- there are no strictly semistable points.

Thus $\left(\mathbb{P}^{n}\right)^{s}=\left(\mathbb{P}^{n}\right)^{s s}$ can be identified with $k^{n} \backslash\{0\}$ and the action of $k^{*}$ becomes

$$
\lambda .\left(x_{0}, \ldots, x_{n-1}\right)=\left(\lambda^{n+1} x_{0}, \ldots, \lambda^{n+1} x_{n-1}\right) .
$$

So

$$
\left(\mathbb{P}^{n}\right)^{s} / k^{*}=\left(\mathbb{P}^{n}\right)^{s s} / / k^{*}=\mathbb{P}^{n-1}
$$

(Of course the action on $k^{n} \backslash\{0\}$ is not quite the standard one but the quotient is the same. In the full theory the description above corresponds to constructing the quotient along with an ample line bundle, which in this case is $\mathcal{O}(n)$.) 


\subsection{Binary forms.}

Example 4.2. We consider the vector space $V_{n+1}$ of homogeneous polynomials in $x_{0}, x_{1}$ of degree $n$, i. e.

$$
V_{n+1}=\left\{f=\sum a_{i} x_{0}^{n-i} x_{1}^{i}: a_{i} \in k\right\} .
$$

A binary form of degree $n$ is an element of $\mathbb{P}\left(V_{n+1}\right)$.

Any binary form can be represented by a non-zero $f \in V_{n+1}$, which can be factorised into linear factors and so determines a set of $n$ points (counted with multiplicities) in $\mathbb{P}^{1}$. Conversely a set of $n$ points (with multiplicities) in $\mathbb{P}^{1}$ determines a binary form. It follows that the natural action of $\operatorname{SL}(2)$ on $\mathbb{P}^{1}$ induces an action on $\mathbb{P}\left(V_{n+1}\right)$. We wish to determine the stable and semistable points for this action using the Hilbert-Mumford criterion.

Any 1-PS of SL(2) is conjugate to one of the form $\lambda_{r}$ for some $r \geq 0$, where

$$
\lambda_{r}(t)=\left(\begin{array}{cc}
t^{r} & 0 \\
0 & t^{-r}
\end{array}\right) .
$$

By Proposition 2.2, a binary form is non-stable (non-semistable) if and only if it is equivalent under the action of $\mathrm{SL}(2)$ to one for which $\mu\left(f, \lambda_{r}\right) \leq 0(<0)$ for some $r$. Now we see by direct computation that

$$
\lambda_{r}(t)\left(\sum a_{i} x_{0}^{n-i} x_{1}^{i}\right)=\sum t^{r(2 i-n)} a_{i} x_{0}^{n-i} x_{1}^{i} .
$$

So the action of $\lambda_{r}$ is diagonal with respect to the obvious basis of $V_{n+1}$, and

$$
\mu=\mu\left(\sum a_{i} x_{0}^{n-i} x_{1}^{i}, \lambda_{r}\right)=r\left(n-2 i_{0}\right)
$$

where $i_{0}$ is the smallest value of $i$ for which $a_{i} \neq 0$. Thus $\mu \leq 0(<0)$ if and only if $a_{i}=0$ whenever $i<\frac{n}{2}\left(i \leq \frac{n}{2}\right)$, i.e. if and only if the point (1:0) occurs as a point of multiplicity $\geq \frac{n}{2}\left(>\frac{n}{2}\right)$ for the given form. Taking account of the action of SL(2), we deduce that a binary form is

- stable if and only if no point of $\mathbb{P}^{1}$ occurs as a point of multiplicity $\geq \frac{n}{2}$;

- semistable if and only if no point of $\mathbb{P}^{1}$ occurs as a point of multiplicity $>\frac{n}{2}$.

Note that, when $n$ is odd, the stable and semistable points coincide, and there is a projective geometric quotient. When $n$ is even, there exist strictly semistable points and the geometric quotient of the stable points is not projective. 


\subsection{Cubic curves.}

Example 4.3. A cubic curve in $\mathbb{P}^{2}$ is defined by a non-zero polynomial

$$
f=\sum a_{i j} x_{0}^{3-i-j} x_{1}^{i} x_{2}^{j}
$$

the summation being taken over all $i, j$ with $i \geq 0, j \geq 0, i+j \leq 3$. The curve determines $f$ up to a scalar multiple, so the set of cubic curves has a natural structure of a projective space $\mathbb{P}^{9}$ with coordinates given by the $a_{i j}$. The group $\operatorname{SL}(3)$ acts naturally through its action on $\mathbb{P}^{2}$.

Before starting on the analysis of stability, we shall summarise information about singular points of the curve defined by $f$. We concentrate on a singular point at $(1: 0: 0)$, since we can translate this information to other points through the action of $\mathrm{SL}(3)$.

- $(1: 0: 0)$ is singular if and only if $a_{00}=a_{10}=a_{01}=0$;

- $(1: 0: 0)$ is a triple point if and only if $a_{00}=a_{10}=a_{01}=a_{20}=a_{11}=a_{02}=0$;

- if $(1: 0: 0)$ is a double point, then the tangents at $(1: 0: 0)$ are the lines defined by the equation

$$
a_{20} x_{1}^{2}+a_{11} x_{1} x_{2}+a_{02} x_{2}^{2}
$$

- in particular, $(1: 0: 0)$ is a double point with a unique tangent $x_{2}=0$ if and only if $a_{00}=a_{10}=a_{01}=a_{20}=a_{11}=0$.

Any 1-PS of SL(3) is conjugate to one of the form $\lambda$, where

$$
\lambda(t)=\left(\begin{array}{ccc}
t^{r_{0}} & 0 & 0 \\
0 & t^{r_{1}} & 0 \\
0 & 0 & t^{r_{2}}
\end{array}\right),
$$

with $r_{0} \geq r_{1} \geq r_{2}$ and $r_{0}+r_{1}+r_{2}=0$. In a similar way to that of Example 4.2, we have

and hence

$$
\lambda(t) . f=\sum t^{(i+j-3) r_{0}-i r_{1}-j r_{2}} a_{i j} x_{0}^{3-i-j} x_{1}^{i} x_{2}^{j}
$$

$$
\mu(f, \lambda)=\max \left\{(3-i-j) r_{0}+i r_{1}+j r_{2}: a_{i j} \neq 0\right\} .
$$

By Proposition 2.2, we know that a cubic curve is non-stable (non-semistable) if and only if it is equivalent under the action of $\mathrm{SL}(3)$ to one for which $\mu(f, \lambda) \leq 0(<0)$ for some $\lambda$ of the above form.

We consider first the condition for semistability. It is easy to check that, if $\mu(f, \lambda)<$ 0 , then

$$
a_{00}=a_{10}=a_{01}=a_{20}=a_{11}=0 .
$$

Conversely, if these conditions hold, and we take $r_{0}=3, r_{1}=-1, r_{2}=-2$, then $\mu(f, \lambda)<0$. Hence $\mu(f, \lambda)<0$ for some $\lambda$ of the above form if and only if the cubic curve has either a triple point at $(1: 0: 0)$ or a double point at $(1: 0: 0)$ with unique tangent $x_{2}=0$. By Proposition 2.2, we deduce that a cubic curve is semistable if and only if it has no triple point and no double point with a unique tangent. 
Next, suppose that $f$ has a singular point; we can assume this to be the point $(1: 0: 0)$, so that $a_{00}=a_{10}=a_{01}=0$. If we take $r_{0}=2, r_{1}=-1, r_{2}=-1$, we get $\mu(f, \lambda) \leq 0$; so $f$ is non-stable.

Conversely, suppose that $\mu(f, \lambda) \leq 0$ for some $\lambda$. Then certainly $a_{00}=a_{10}=0$. If $a_{01} \neq 0$, then

$$
\mu(f, \lambda) \leq 0 \Rightarrow 2 r_{0}+r_{2} \leq 0 \Rightarrow r_{1}=r_{0}, r_{2}=-2 r_{0} .
$$

For these values of the $r_{i}$, we have

$$
\mu(f, \lambda)=\max \left\{(3-3 j) r_{0}: a_{i j} \neq 0\right\}
$$

so $\mu(f, \lambda) \leq 0$ if and only if $a_{i 0}=0$ for all $i$. This implies that $f=x_{2} f^{\prime}$ for some $f^{\prime}$ of degree 2 ; hence $f$ is singular at every point for which $x_{2}=f=0$. Thus in all cases $f$ has a singular point, and we conclude that a cubic curve is stable if and only it is non-singular.

To summarise, the stable cubic curves are the non-singular ones, while the strictly semistable ones have one or more double points with distinct tangents. In fact, there are just three types of strictly semistable cubic curves, each forming a single orbit.

- nodal cubics, i. e. irreducible cubics possessing a double point with distinct tangents;

- cubics which degenerate into a conic and a line not tangent to it;

- cubics which degenerate into three non-concurrent lines. 